\title{
High-energy exclusive leptoproduction of vector mesons *
}

\author{
Thierry Gousset \\ Institut für Theoretische Physik, Philosophenweg 19, D-69120 Heidelberg \\ NIKHEF, P.O. Box 41882, NL-1009 DB Amsterdam
}

\begin{abstract}
The physics of diffractive vector meson production in virtual photon nucleon scattering at NMC energies is reviewed. A particular attention is paid to the physical aspects of the reaction and how they influence the observables. The reaction is a good probe to investigate both soft exchange mechanisms and hadronic wave functions. Extension to either HERA or ELFE kinematics is sketched out.
\end{abstract}

\section{Space-time aspect of the reaction}

The reaction of interest is, within the one-photon exchange approximation,

$$
\gamma^{*}+p \rightarrow V+p
$$

where $V$ is a vector meson $(\rho, \phi, J / \psi$, etc.). It is depicted in Fig. 1. The present review focuses on vector meson production off a proton target. The extension to nuclear targets is of great importance to observe the phenomenon of color transparency. It has also been discussed during the meeting [1].

We are interested in high-energy processes, i.e. when the center of mass energy $W=\sqrt{(q+p)^{2}}$ is of the order of $10 \mathrm{GeV}$ or more, and in the low momentum transfer region, $-t=-\left(q-q^{\prime}\right)^{2}<1 \mathrm{GeV}^{2}$. In this kinematical domain the strong interaction is predominantly mediated by the pomeron. What are the precise properties of the pomeron and how such an object can emerge from QCD is still under debate, hence the interest of the field. In order to have a simple behavior of the photon, we will also limit ourselves to the region of large $Q^{2}=-q^{2}$, say $Q^{2}>2 \mathrm{GeV}^{2}$, but small $x_{B}=Q^{2} / 2 p . q<0.1$.

\footnotetext{
^ to appear in the proceedings of the workshop ELFE at DESY
} 


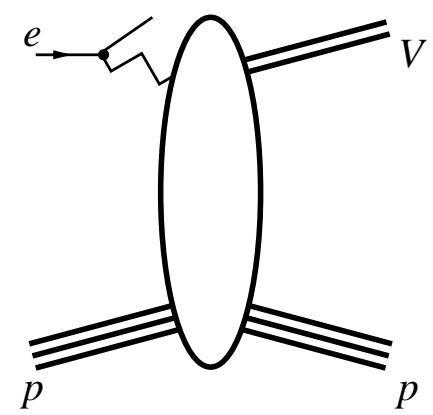

Fig. 1. The reaction $\gamma^{*}+p \rightarrow V+p$. The initial photon 4-momentum is denoted with $q$, the initial nucleon momentum with $p$, and the equivalent final states with $q^{\prime}$ and $p^{\prime}$.

The fact that reactions induced by a photon, either real or virtual, can help to get insights into the diffractive domain of strong interaction is due to the tendency of the photon to split up into partonic components [2]. To see this, let us consider, in the proton rest frame, a photon flying in the $z$-direction so that $q=\left(\nu, \mathbf{0}_{T}, \sqrt{\nu^{2}+Q^{2}}\right)$. The two possible time-ordered configurations are depicted in Fig. 2, where:

- (a) the photon directly attaches to a proton constituent;

- (b) the photon fluctuates into a $q \bar{q}$-pair before reaching the proton.
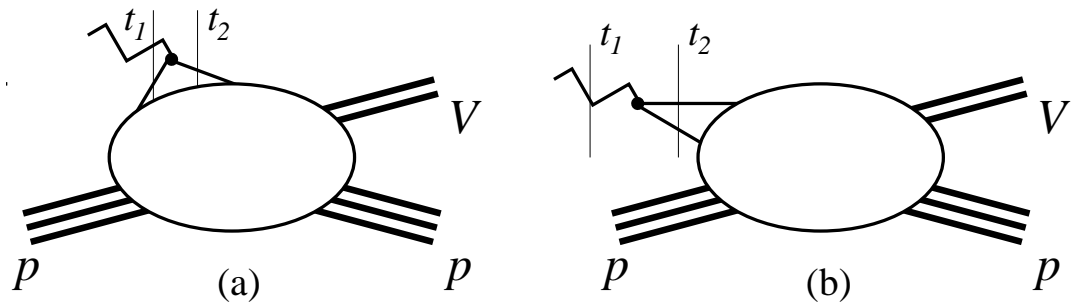

Fig. 2. The two possible time-ordered configurations $\left(t_{1}<t_{2}\right)$.

The strength of each of these possibilities is a matter of energy denominators between initial and intermediate states. In Fig. 2(a), the quark energy is $E=$ $\left(K_{z}^{2}+\mathbf{K}_{T}^{2}+m_{q}^{2}\right)^{1 / 2}$ before the photon vertex and $E^{\prime}=\left(\left(q_{z}+K_{z}\right)^{2}+\mathbf{K}_{T}^{2}+m_{q}^{2}\right)^{1 / 2}$ after. The magnitude of the initial quark momentum components, $K_{z}$ and $\mathbf{K}_{T}$, is fixed by the proton wavefunction and is $O\left(1 / R_{p}\right)$, with $R_{p}$ the proton radius. This leads to the estimate $\Delta E_{a}=E+\nu-E^{\prime}=O\left(1 / R_{p}\right)$. In Fig. 2(b), we have $E_{q}=\left(z^{2}\left(\nu^{2}+Q^{2}\right)+\mathbf{k}_{T}^{2}+m_{q}^{2}\right)^{1 / 2}$ for the quark, where $z=k_{z} / q_{z}$ is the longitudinal fraction it carries. To get the antiquark energy, $E_{\bar{q}}$, we replace $z$ by $1-z$ in $E_{q}$. The magnitude of $\mathbf{k}_{T}$ and $z$ is now determined by the vector meson wave function. This leads to

$$
\left(\Delta E_{b}\right)^{-1}=\frac{2 \nu}{Q^{2}+\frac{\mathbf{k}_{T}^{2}+m_{q}^{2}}{z(1-z)}} \approx\left(x_{B} M_{p}\right)^{-1}
$$


which dominates $\left(\Delta E_{a}\right)^{-1}$ in the small $x_{B}$ region we are considering.

This can be reformulated by saying that the $q \bar{q}$-fluctuation has a lifetime or coherence length

$$
l_{c}=\left(E_{q}+E_{\bar{q}}-\nu\right)^{-1} \approx\left(x_{B} M_{p}\right)^{-1},
$$

much larger than the interaction time, i.e. the proton radius, at very small $x_{B}$. As a consequence, the proton practically always sees the photon as a quarkgluon wave packet in this regime. In the proton rest frame (and in the center mass frame as well), a simple physical picture of the process emerges where the reaction is a three-step process:

- the photon turns into a quark-gluon state,

- this state then scatters softly on the proton constituents,

- before it forms the final vector meson.

From this physical evolution of the reaction, we deduce that experimental studies of vector meson electroproduction help us to learn about both hadronic wavefunctions and soft exchange mechanism ("pomeron exchange").

To be more specific, the amplitude of the process can be written as

$$
\mathcal{M}\left(\gamma^{*}+p \rightarrow V+p\right)=\int d z d^{2} \mathbf{r} \psi_{\gamma^{*}}(z, \mathbf{r}) \mathcal{M}(q \bar{q}+p \rightarrow q \bar{q}+p) \psi_{V}^{*}(z, \mathbf{r})
$$

where $\psi_{\gamma^{*}}$ and $\psi_{V}$ are the photon and vector meson light-cone wave function, respectively. $\mathcal{M}(q \bar{q}+p)$ is the interaction amplitude between the $q \bar{q}$-dipole, with transverse extension $\mathbf{r}$, and the proton. It can be in turn expanded in terms of the initial and final proton wavefunctions and a fully microscopic interaction amplitude at the quark-gluon level. The process is thus similar to the high-energy elastic collisions of a meson with a proton, in which case the amplitude reads

$$
\mathcal{M}(M+p \rightarrow M+p)=\int d z d^{2} \mathbf{r} \psi_{M}(z, \mathbf{r}) \mathcal{M}(q \bar{q}+p \rightarrow q \bar{q}+p) \psi_{M}^{*}(z, \mathbf{r}),
$$

but the photon virtuality $Q^{2}$ and polarization (either longitudinal or transverse) provide extra degrees of freedom in addition to the center of mass energy $\sqrt{s} \equiv W$ and momentum transfer $t$.

The phenomenology associated with the photon degrees of freedom is determined from its wavefunction. The simplest $q \bar{q}$-component can be easily computed in perturbation theory. Its main property is that large transverse distances are suppressed by a factor $e^{-\varepsilon r}$ where $\varepsilon=\sqrt{z(1-z) Q^{2}+m_{q}^{2}}$. In vector meson production, it is actually the wave function overlap, $\psi_{\gamma^{*}} \psi_{V}^{*}$, which matters, so that the end-point region in $z$ is relatively unimportant, and the transverse distance probed diminishes with increasing $Q^{2}$. Further- 
more, it turns out that this overlap depends on the photon polarization, the important differences being that

$$
\left.\psi_{\gamma^{*}} \psi_{V}^{*}\right|_{L} \propto z(1-z) Q,\left.\quad \psi_{\gamma^{*}} \psi_{V}^{*}\right|_{T} \propto \varepsilon r
$$

from which we can infer that the longitudinal transition dominates the transverse one at large $Q^{2}$.

\section{The Donnachie-Landshoff Model [3]}

A phenomenological step towards the understanding of soft collisions can be accomplished by extracting their universal characteristics. This can be done by discussing the model of Donnachie and Landshoff. The model is based on a Regge approach and aims at describing every high-energy elastic collisions with a few ingredients. Despite (thanks to?) its simplicity, it gives a reasonable account of the present hadron-hadron elastic scattering data.

The model has a diagrammatic formulation and the relevant diagram for pionproton collision at large center of mass energy, $\sqrt{s}$, and small momentum transfer, $t$, is shown in Fig. 3.

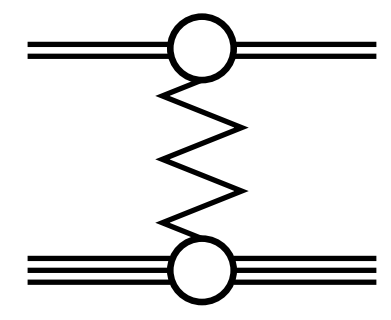

Fig. 3. Diagram for $\pi-p$ elastic scattering.

In Fig. 3, the exchanged object is a pomeron to which one associates the factor

$$
2 i\left(\frac{s}{s_{0}}\right)^{\alpha_{0}+\alpha^{\prime} t}
$$

where the pomeron intercept, $\alpha_{0}$, is observed to be $\alpha_{0} \approx 1.08$ and the slope $\alpha^{\prime} \approx 0.25 \mathrm{GeV}^{-2}$. Other objects (reggeons) can be exchanged in the reaction. Their contributions are however subleading in energy, i.e. suppressed by some powers of $1 / s$, and will be ignored for simplicity.

The second necessary ingredient in Fig. 3 is the hadron-pomeron vertex. Phenomenologically, one obtains a good description assuming that the pomeron couples to quarks like a photon. Of course, it does not interact with the individual quarks via their electromagnetic charge but rather couples to each 
constituent quark with a strength $\beta_{0}$. The pion-pomeron and proton-pomeron coupling are then

$$
(\pi-P-\pi)=2 \beta_{0} F_{\pi}(t), \quad(p-P-p)=3 \beta_{0} F_{1}(t)
$$

where the $t$-dependence of the vertex is given by the hadron leading form factor.

As already stated, this model provides a good description of total cross sections which are related to the elastic amplitude through the optical theorem

$$
\sigma(s)=\frac{1}{s} \operatorname{Im} \mathcal{M}(s, t=0),
$$

with a universal "slow" rise $s^{0.08}$ in the $\sim 100 \mathrm{GeV}$ region. Including the nextto-leading trajectory, the model provides good fits down to $\sqrt{s}=5 \mathrm{GeV}$ for $p+p, \bar{p}+p, \pi+p, K+p, \gamma+p[4]$.

The fact that the pomeron counts the number of constituents inside the hadron is called quark additivity. With this property, wave function effects expected from Eq. (1) are hidden inside the form factor that enters into the hadronpomeron coupling.

Let us now return to the exclusive electroproduction of $\rho$-mesons. In the Donnachie-Landshoff model, the relevant graph is depicted in Fig. 4.

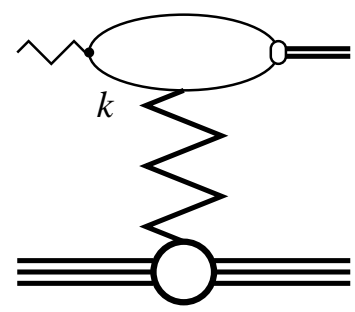

Fig. 4. Diagram for exclusive $\rho$-electroproduction at large $Q^{2}$.

The only difference with the diagram describing pion-proton scattering, Fig. 3, is that the pion-pomeron vertex is now replaced by a quark-loop. Assuming that a non-relativistic description of the $\rho$-meson suffices to evaluate the quark-loop, one sees that the quark with momentum $k$ is far off-shell with $k^{2} \approx-Q^{2} / 2$. Under this circumstance where small transverse distances are being probed, the quark-pomeron interaction can no longer be considered as pointlike and the strength $\beta_{0}$ must be replaced by a decreasing interaction, $\beta_{0} f\left(k^{2}\right)$, with $f\left(k^{2}\right)$ a pomeron form factor. The deep reason for this phenomenological introduction of a form factor is that the QCD interaction of a color neutral dipole of size $r$ vanishes like $r^{2}$ at small $r$. This colour transparency phenomenon implies that, at small transverse distances, quark addi- 
tivity cannot hold in QCD. This aspect needs not be present in hadron-hadron soft interaction where the off-shellness of quarks, $k^{2}$, is limited by hadronic wavefunctions and therefore $f\left(k^{2}\right)$ may always stay close to 1 .

We thus get for the photon-pomeron-rho vertex

$$
2 \beta_{0} f\left(k^{2}\right) \frac{e f_{\rho} M_{\rho}}{2 k^{2}}
$$

The factor $k^{2}$ in the denominator comes from the quark propagator in Fig. 4. Within the non-relativistic description of the $\rho$-meson, the remaining part of the quark loop in Fig. 4 is simply related to the $\rho$ decay constant, $f_{\rho}$, defined as

$$
\left\langle 0\left|J^{\mu}(0)\right| \rho(q, h)\right\rangle=e f_{\rho} M_{\rho} \varepsilon^{\mu}(q, h)
$$

i.e. to the $\rho$ wave function at the origin.

The differential cross section is obtained by putting all the factors together and squaring. With these inputs, the model provides a good description for the $Q^{2}$-behavior of the production cross section observed by EMC and NMC for the $\rho$-meson [3]. From Eq. (3), an energy dependence $\sim W^{0.32}$ is expected. This is at variance with the observed rising at HERA. I will return to this point in section 5 .

\section{Towards a microscopic description of the pomeron}

Soon after the formulation of QCD, Low and Nussinov have proposed that pomeron exchange corresponds to soft gluon exchanges [5]. The problem is then to treat the gluon dynamics in the nonperturbative domain of QCD. Here I consider the stochastic vacuum model [6]. This model and its application to high-energy scattering has been reviewed during the First ELFE Summer School [7].

The model accomodates the non-trivial structure of the QCD vaccuum. Evidence for a non-trivial structure (as compared to a perturbative vacuum) is given by the sizeable value of the gluon condensate

$$
\left\langle g^{2} F F\right\rangle=\left\langle 0\left|\frac{g^{2}}{4 \pi^{2}} F_{\mu \nu}^{a}(x) F^{a \mu \nu}(x)\right| 0\right\rangle \approx(0.4 \mathrm{GeV})^{4}
$$

when compared to the QCD scale $\Lambda \approx 0.2 \mathrm{GeV}$. Within the model, the QCD vacuum is considered as a superposition of configurations where the gluon 
field strength condenses into domains (analogously to the magnetic field in a ferromagnet).

The basic ingredient in the model is a gluon field strength two-point correlator. In order to define a gauge invariant correlator, one has to introduce the modified gluon field strength $F_{\mu \nu}(x, \omega)$ which is obtained from the field strength at point $x$ by parallel transporting the colour content to a reference point $\omega$

$$
F_{\mu \nu}(x, \omega)=\Phi^{-1}(x, \omega) F_{\mu \nu}(x) \Phi(x, \omega),
$$

with $\Phi(x, \omega)=P \exp \left[-i g \int_{\omega}^{x} A d z\right]$. Assuming that the main features of the correlator $\left\langle 0\left|F_{\mu \nu}(x, \omega) F_{\rho \sigma}(y, \omega)\right| 0\right\rangle$ do not depend crucially on the choice of the reference point $\omega$ we obtain for the dependence on $z=x-y$ the most general form

$$
\begin{aligned}
& \left\langle g^{2} F_{\mu \nu}^{c}(x, \omega) F_{\rho \sigma}^{d}(y, \omega)\right\rangle=\frac{\delta^{c d}}{N_{c}^{2}-1} \frac{\left\langle g^{2} F F\right\rangle}{12}\left\{\kappa\left(\eta_{\mu \rho} \eta_{\nu \sigma}-\eta_{\mu \sigma} \eta_{\nu \rho}\right) D\left(z^{2} / a^{2}\right)\right. \\
& \left.+(1-\kappa) \frac{1}{2}\left[\partial_{\mu}\left(z_{\rho} \eta_{\nu \sigma}-z_{\sigma} \eta_{\nu \rho}\right)+\partial_{\nu}\left(z_{\sigma} \eta_{\mu \rho}-z_{\rho} \eta_{\mu \sigma}\right)\right] D_{1}\left(z^{2} / a^{2}\right)\right\} .
\end{aligned}
$$

The correlator incorporates the gluon condensate at $x=y$ and the fall-off of the correlation of two field strengths at large distances. This fall-off occurs on a typical correlation length, $a$, the typical size of the domain, which turns out to be about $0.3 \mathrm{fm}$. The correlator $D$ is specific for a non-Abelian gauge theory since the homogeneous Maxwell equations

$$
\epsilon^{\mu \nu \rho \sigma} \partial_{\nu} F_{\rho \sigma}=0
$$

allow only the tensor structure associated with $D_{1}$ in Eq. (5), i.e. $\kappa=0$ in an Abelian theory without magnetic monopoles.

The model leads to linear confinement, i.e. the Wison loop satisfies an area law for large contours, only if $\kappa \neq 0$. In this approach, confinement is then explicitely due to the non-Abelian nature of QCD. Notice that lattice simulations support the non-Abelian character of the correlator Eq. (5) by giving $\kappa \approx 0.74[8]$.

The high-energy scattering of two color singlet dipoles $q_{1} \bar{q}_{1}$ and $q_{2} \bar{q}_{2}$ can be treated analogously to the situation of heavy quarks encountered in the Wilson area law. In the center of mass frame, each quark (or antiquark) travels with nearly the speed of light along some $z$-direction for $q_{1} \bar{q}_{1}$ or the opposite for $q_{2} \bar{q}_{2}$. During their travel, quarks interact with the vacuum field. This interaction shows up as a non abelian phase [9]

$$
V=P \exp \left[-i g \int_{\Gamma} A d z\right]
$$


where $\Gamma$ is the classical path of the quark (an antiquark gets a similar phase but its path is backward oriented). For dipole 1 , the quark phase, $V_{1}$, together with the antiquark phase, $V_{\overline{1}}$, and the two gauge links entering the definition of the initial and final state dipole give rise to a Wilson loop $W_{1}$. The resulting dipole-dipole interaction is then the expectation value of the product of the two loops $W_{1}$ and $W_{2}$.

In the model of the stochastic vacuum, this expectation value is related to the two-point correlator [10]. The two components of the correlator in Eq. (5) gives rise to distinct types of interaction. This is illustrated in Fig. 5 where the interaction amplitude between a large dipole target oriented along a given $x$-axis and a small dipole probe is plotted as a function of the impact position for both (a) the "non-confining" case, $\kappa=0$, and (b) the "purely confining" case, $\kappa=1$. For simplicity a sum over the orientation of the probe has been carried out.
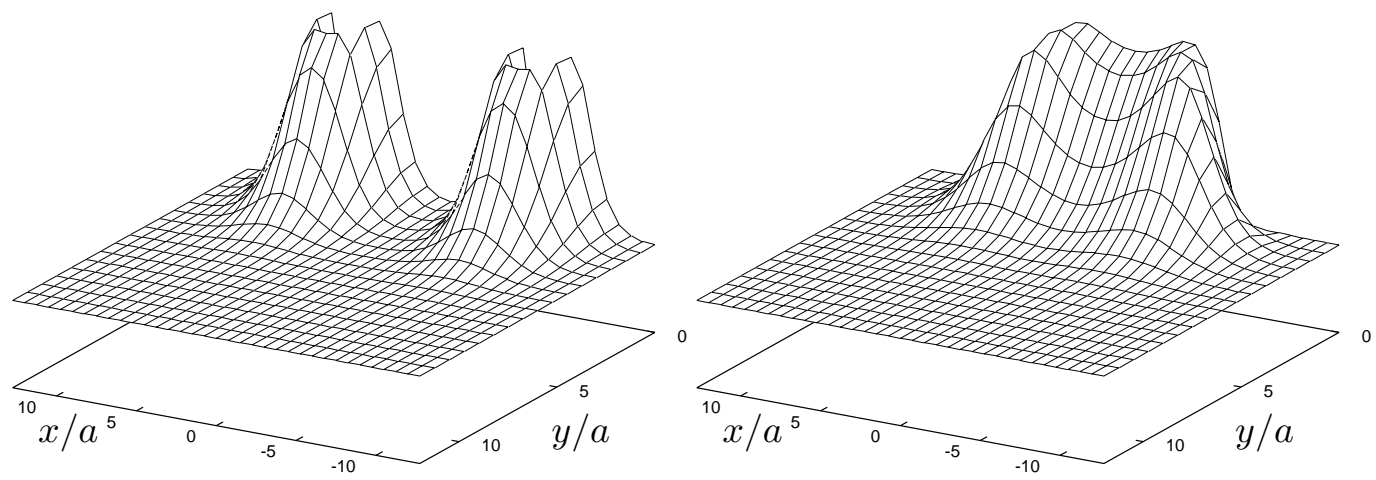

Fig. 5. (a) Color interaction amplitude for $\kappa=0$ as a function of the impact position between the two dipoles. Dipole 1 has a transverse size $r_{1}=a$ and an average over its orientation is understood. Dipole 2 has a transverse size $r_{2}=12 a$ and lies along the $x$-axis.

(b) Color interaction amplitude for $\kappa=1$.

The non-confining term gives rise to a quark-quark interaction, where color charges only interact if their trajectories enter in a common domain of the vacuum structure. The confining term gives a string-string interaction where the color dipoles (connected by their strings) interact as whole objects rather than as isolated endpoints. We notice that quark additivity arises from the non-confining part of the interaction provided hadrons are much bigger than the domain size, but that it does not if the confining term is important.

From the nonperturbative scattering amplitude of color dipoles with fixed lengths $r_{1}$ and $r_{2}$, a valence quark picture can be constructed by distributing the positions of the end-points of the strings according to hadronic wave functions [10]. Integrating out the nucleon constituent degrees of freedom leads to our model amplitude for the dipole-proton interaction amplitude to be inserted 
in Eq. (1).

\section{Comparison with experiment}

From the dipole-proton interaction amplitude just discussed and with the perturbative photon wavefunction and a model wave function to describe the vector meson, one can compute the differential cross section for either a longitudinal or a transverse polarization of the photon [11]. The discussion of our results for these quantities gives me the opportunity to show the present experimental status at $\mathrm{NMC}$ energies, i.e. $W \approx 10-20 \mathrm{GeV}$.

Let us first compare with the integrated cross section for $\rho$ and $J / \psi$-production. The experimental results come in the combination $\sigma_{\exp }=\epsilon \sigma_{L}+\sigma_{T}$, where $\epsilon$ is the rate of longitudinally polarized photons. The results as a function of $Q^{2}$ are show in Fig. 6.
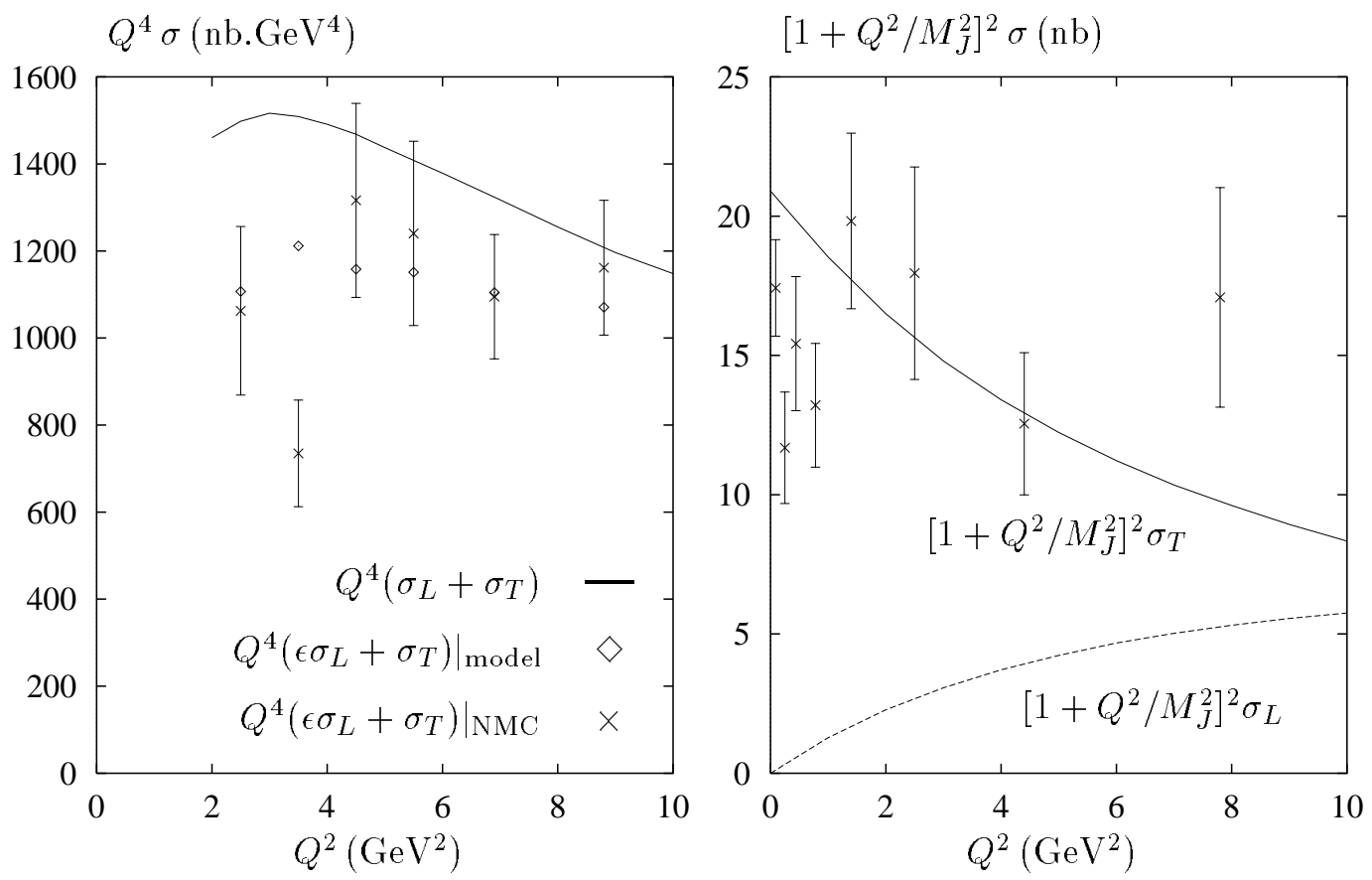

Fig. 6. (a) The scaled cross section $Q^{4} \sigma\left(Q^{2}\right)$ for $\rho$-production in $n b \cdot \mathrm{GeV}^{4}$. The crosses are the NMC-results [12] and the diamonds represent our prediction for the quantity $Q^{4}\left(\epsilon \sigma_{L}+\sigma_{T}\right)$ with the experimental polarization rate of NMC.

(b) $J / \psi$-production cross section for longitudinal and transverse polarizations. To compare with EMC-data [13], one has to combine these two cross sections into $\sigma=\epsilon \sigma_{L}+\sigma_{T}$ with the polarization rate measured by EMC, $\epsilon \approx 0.7$.

The model parameters, $\left\langle g^{2} F F\right\rangle$ and $a$, were adjusted to fit the proton-proton 
total cross section and elastic slope. Keeping them fixed, the magnitude of our cross sections depends on the meson wave function parameters. Once the value of the wave function at the origin is fixed to fit the meson decay constant, see Eq. (4), the most important ingredient is the transverse extension parameter for light quark systems and the quark mass when heavy quarks are involved. With the shrinkage of the photon, this influence decreases with increasing $Q^{2}$. The exact $z$-dependence of the wave function also affects the overall magnitude of the cross section.

The $Q^{2}$-behavior of specific observables, such as the ratio of longitudinal to transverse cross sections, $R\left(Q^{2}\right)=\sigma_{L} / \sigma_{T}$, offers possibilities to distinguish between different models. The ratio is plotted in Fig. 7(a). For large $Q^{2}, R=$ $O\left(Q^{2}\right)$ but this behavior is not yet reached in the intermediate range where $R$ grows slower than $Q^{2}$.
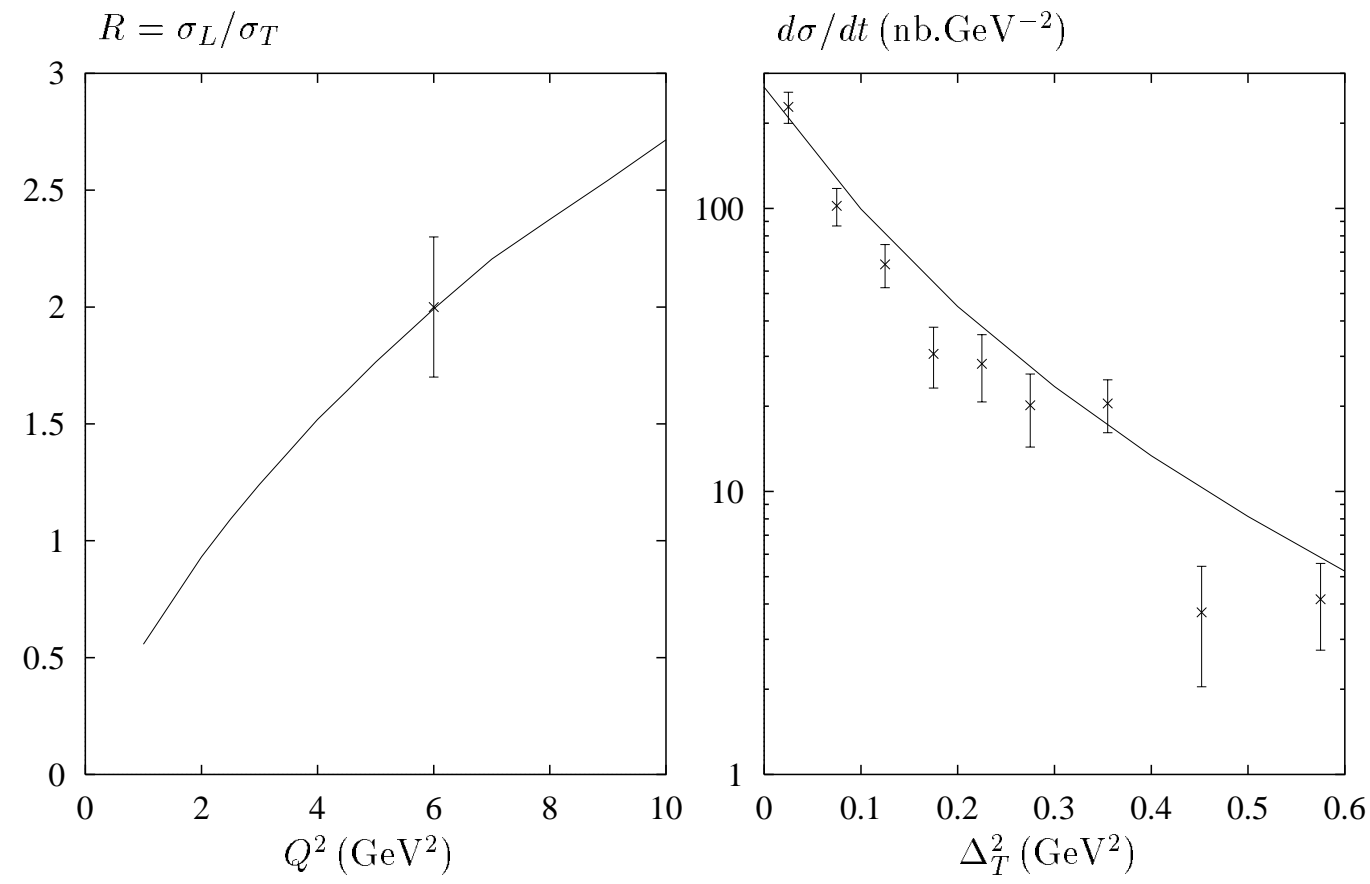

Fig. 7. (a) The ratio for longitudinal to transverse cross section for $\rho$-production. The data point is from Ref. [12]. Other data compare well within errors but they either are far outside the $10-20 \mathrm{GeV}$ range or have large errorbars.

(b) The differential cross section, $d \sigma / d t\left(\Delta_{T}^{2}\right)$, for $\gamma^{*}+p \rightarrow \rho+p$ at $Q^{2}=6 \mathrm{GeV}^{2}$. Data are from Ref. [12].

Another important check is provided by looking at the $t$-dependence of the differential cross section. We show our result for $\epsilon d \sigma_{L} / d t+d \sigma_{T} / d t$ versus $\Delta_{T}^{2}$ at $6 \mathrm{GeV}^{2}$ in Fig. 7(b) and compare it to the NMC points for the deuteron outside of the coherent production region [12]. Notice also that there is a non trivial $Q^{2}$-dependence of the slope $B=d \ln (d \sigma / d t) / d t$ which comes with the 
slowly shrinking transverse size of the photon as $Q^{2}$ grows.

\section{$5 \quad$ Energy dependence}

The pomeron phenomenology extracted from hadron-hadron scattering as in section 2 predicts for the exclusive vector meson electroproduction cross section a universal energy dependence $\sim W^{0.32}$ for large $W$. According to recent HERA results, the energy dependence at large $Q^{2}$ is however much more pronounced than such a "soft" behavior. The observed trend of the data is shown in Fig. 8.

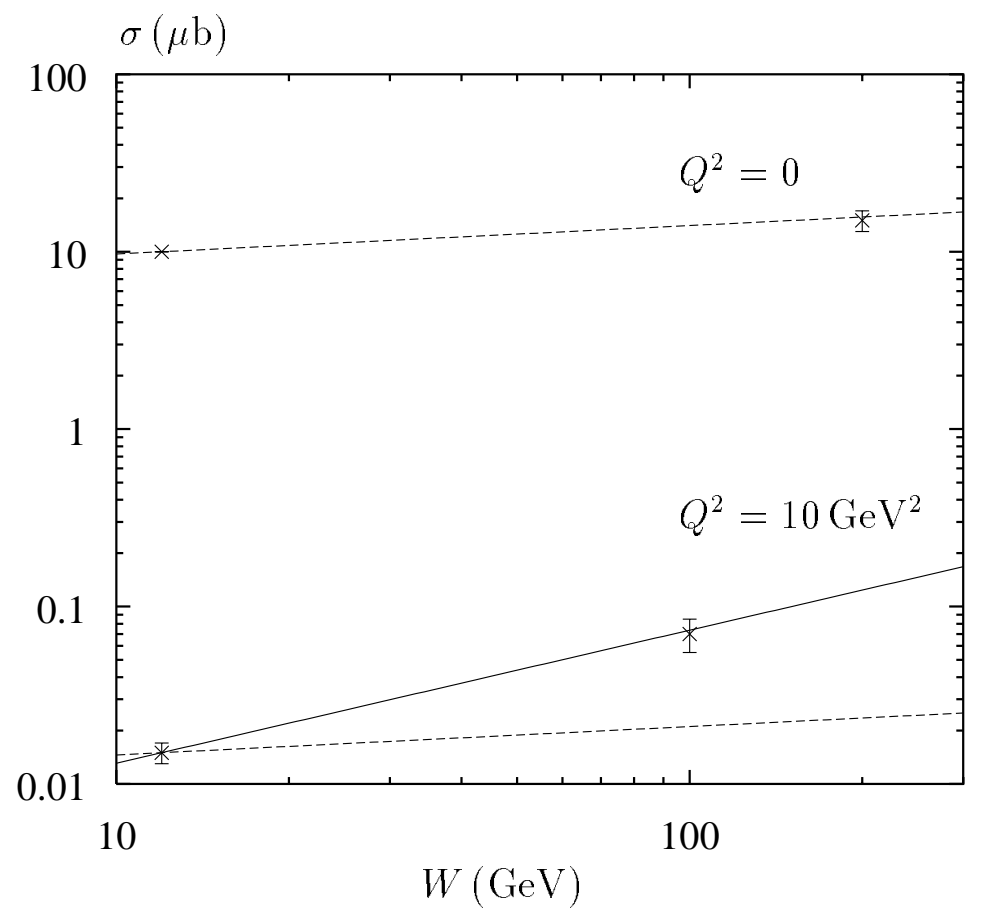

Fig. 8. Photoproduction and electroproduction at $Q^{2}=10 \mathrm{GeV}^{2}$ of $\rho$-mesons. The energy rise observed in photoproduction is compatible with the soft pomeron phenomenology. A quite different behavior is observed at large $Q^{2}$

Such a behavior has to be compared with the energy-dependence observed for the total photon-proton cross section, the latter also showing a weak dependence, $W^{0.16}$, for $Q^{2}=0$, and a strong one at large $Q^{2}$. The physics driving the rise of $F_{2}\left(x, Q^{2}\right)$, which is related to the total cross section through

$$
F_{2}\left(x, Q^{2}\right)=\frac{Q^{2}}{\pi e^{2}}\left(\sigma_{T}+\sigma_{L}\right)
$$

towards small $x_{B} \approx Q^{2} / W^{2}$ (i.e., at fixed $Q^{2}$, towards large energy) may 
then explain what occurs in electroproduction. $\square$ In the same spirit, it should be noted that the energy dependence in $J / \psi$-photoproduction is similar to large $Q^{2} \rho$-production. A possible explanation for this similarity is that the typical transverse distances involved in both cases are the same. This can be deduced from the expression of the "photon size" defined in section 1 , $\varepsilon=\left[z(1-z) Q^{2}+m_{q}^{2}\right]^{1 / 2}$. In the $J / \psi$-case and at $Q^{2}=0$, the presence of the large $c$-quark mass leads to a typical transverse size probed as small as in the light quark case at some large $Q^{2}$ (a quantitative estimate gives $Q^{2} \approx M_{J / \psi}^{2} \approx 10 \mathrm{GeV}^{2}$ ). A study of the energy dependence in vector meson electroproduction in which the above connections appear naturally is given in Ref. [14].

One can estimate the $t \rightarrow 0$ limit of the amplitude $\mathcal{M}(q \bar{q}+p \rightarrow q \bar{q}+p)$ appearing in Eq. (1) for small transverse distances. It is related, through the optical theorem ${ }^{2}$

$$
\mathcal{M}(s, r, t=0)=i s \sigma^{\mathrm{tot}}(s, r)
$$

to the total cross section of a small $q \bar{q}$-dipole, with transverse extension $r$, scattering on a proton. This in turn can be evaluated

$$
\sigma^{\mathrm{tot}}(s, r)=\frac{\pi^{2}}{3} \alpha_{S} x G_{p} r^{2}
$$

where $G_{p}$ is the gluon density in the proton. Replacing this in Eq. (1), one gets for the dominant part at large $Q^{2}[15,16]$

$$
\mathcal{M}(s, t=0)=i s \frac{\pi^{2}}{3} \alpha_{S} x G_{p} \frac{4 e f_{V} \int_{0}^{1} d z \frac{\varphi_{V}(z)}{z(1-z)}}{Q^{3} \int_{0}^{1} d z \varphi_{V}(z)} .
$$

Let us first notice that, the leading transition at large $Q^{2} \gg M_{V}^{2}$ is that of a longitudinal photon turning into a longitudinal vector meson. Other transition amplitudes are suppressed by some power of $1 / Q$ and are asymptotically negligible in the cross section.

In the limit of large $Q^{2}$, the meson wave function is probed only at 0 -transverse distance, i.e. it is the distribution amplitude, $\varphi_{V}(z)=\psi_{V}\left(z, r_{T}=0\right)$, of the

1 A closer look to transverse distances involved shows that exclusive vector meson production compares better to the longitudinal cross section $\sigma_{L}$ than to the sum $\sigma_{T}+\sigma_{L}$

2 the real part of $\mathcal{M}$ can be neglected 
vector meson which matters in the meson wave function together with the meson decay constant $f_{V}$. Actually, it is only the quantity

$$
\eta_{V}=\int_{0}^{1} d z \frac{\varphi(z)}{2 z(1-z)}
$$

for a normalized $\varphi, \int_{0}^{1} d z \varphi(z)=1$, which enters in the expression of the scattering amplitude. The distribution amplitude also occurs in exclusive reactions at large momentum transfer [17], but in general inside different integrals. For the purpose of extracting distribution amplitude from experiments, the present reactions turn out to be complementary to hard exclusive ones.

One of the difficulties in the above derivation is to find how are the arguments $x$ and $\mu^{2}$ entering in the gluon density $x G_{p}\left(x, \mu^{2}\right)$ related to the physical $x_{B}$ and $Q^{2}$. One of the problem is that the above amplitude is explicitely computed at $\Delta=0$, a limit which turns out to be unphysical since the vector meson and the photon do not have the same mass. This difficulty can be circumvented by introducing off-forward parton densities [18].

The remaining $t$-dependence of the amplitude has a soft origin and is not accessible via a perturbative treatment. Its description requires a non-perturbative approach such as the one presented in section 3.

\section{ELFE regime}

The experimental accuracy reached so far has revealed basic facts about the physics at work in vector meson production at small momentum transfer. However quantitative tests which are necessary to disentangle the various aspects of the reaction seem only feasible with high-luminosity facilities.

The various approaches described above rely on high-energy approximation. At ELFE, the maximum center of mass energy is $W \approx 5 \mathrm{GeV}$, a value which lies just at the low end of our high-energy domain. From the Regge phenomenology point of view, exchanges of reggeons other than the pomeron are important in this energy domain. This aspect makes the microscopic description of the process much more difficult. To avoid this complication, it is interesting to focus on $\phi$ and $J / \psi$-production because the coupling of these particles to non-vacuum trajectories is strongly suppressed.

With the limitation $x_{B} \leq 0.1$, the range of $Q^{2}$ is restricted to $Q^{2} \leq 3 \mathrm{GeV}^{2}$, that is with ELFE one just reaches the "hard" region where a simple $q \bar{q}$ description of the photon may be sufficient to describe the process. An under- 
standing of the small $Q^{2}$ region is necessary. The $Q^{2} \rightarrow 0$ limit is commonly described through the vector meson dominance of the photon and, within the Donnachie-Landshoff model, it gives a reasonable description of $\rho$ and $\phi$-production.

Finally two very interesting extensions have not been discussed here. These are, on the one hand, the study of the reaction for nuclear targets instead of the nucleon and, on the other hand, the transition from low to high momentum transfer.

\section{Acknowledgements}

I thank Rainer Jakob for his careful reading of the manuscript. Part of this review describes a work carried out with H.G. Dosch, G. Kulzinger and H.J. Pirner. This work was supported by the Federal Ministry of Education, Science, Research and Technology (BMBF) under grant no. 06 HD 742.

\section{References}

[1] see the contributions to the color transparency session by B. Kopeliovich and G. Piller.

[2] T.H. Bauer et al., Rev. Mod. Phys. 50 (1978) 261.

[3] A. Donnachie and P.V. Landshoff, Phys. Lett. B 185 (1987) 403; ibid. 348 (1995) 213.

[4] Review of Particle Physics, Phys. Rev. D 54 (1996) 191.

[5] F.E. Low, Phys. Rev. D 12 (1975) 163; S. Nussinov, Phys. Rev. Lett. 34 (1975) 1286.

[6] H.G. Dosch, Phys. Lett. B 190 (1987) 177; H.G. Dosch and Y.A. Simonov, ibid. 205 (1988) 339.

[7] O. Nachtmann, in: Proceedings of the First ELFE Summer School on confinement physics, eds. S.D. Bass and P.A.M. Guichon (Editions Frontières, Gif sur Yvette, 1996).

[8] A. Di Giacomo and H. Panagopoulos, Phys. Lett. B 285 (1992) 133.

[9] O. Nachtmann, Ann. Phys. 209 (1991) 436.

[10] H.G. Dosch, E. Ferreira and A. Krämer, Phys. Rev. D 50 (1994) 1992.

[11] H.G. Dosch et al., hep-ph/9608203, submitted to Phys. Rev. D. 
[12] NMC, M. Arneodo et al., Nucl. Phys. B429 (1994) 503.

[13] EMC, J.J. Aubert et al., Nucl. Phys. B213 (1993) 1.

[14] J. Nemchick et al., Phys. Lett. B 374 (1996) 199, and references therein.

[15] M.G. Ryskin, Z. Phys. C 57 (1993) 89.

[16] S.J. Brodsky et al., Phys. Rev. D 50 (1994) 3134.

[17] S.J. Brodsky and G.P. Lepage, in: Perturbative QCD, ed. A.H. Mueller (World Scientific, Singapore, 1989).

[18] A.V. Radyushkin, Phys. Lett. B 385 (1996) 333. 